\title{
LA EXPERIENCIA DEL TRAYECTO CURRICULAR INTEGRADOR TRABAJO SOCIAL COMUNITARIO EN LA UNIVERSIDAD NACIONAL DE AVELLANEDA: APUNTES SOBRE UN PUNTO DE PARTIDA
}

DOI: $d x . d o i . o r g / 10.18616 /$ inser07

Rodrigo Ávila Huidobro ${ }^{1}$

Liliana Elsegood ${ }^{2}$

Ignacio Garaño ${ }^{3}$

Facundo Harguinteguy ${ }^{4}$

\section{INTRODUCCIÓN}

\section{PENSAR LA CURRICULARIZACIÓN DE LA EXTENSIÓN EN LA TRADICIÓN DE LA UNIVERSIDAD NACIONAL ARGENTINA}

En el presente capítulo daremos cuenta de algunas tensiones que, creemos, atraviesan el proceso de curricularización de la extensión en nuestra universidad: estamos hablando por un lado de la obligatoriedad de la extensión universitaria en las currículas de las distintas carreras, reconociendo a la misma como parte del proceso formativo, y, por otra parte, de

\footnotetext{
${ }^{1}$ Antropólogo. Docente, investigador y extensionista de la Universidad Nacional de Avellaneda. ${ }^{2}$ Psicóloga. Docente, investigadora y extensionista de la Universidad Nacional de Avellaneda. ${ }^{3}$ Antropólogo. Docente, investigador y extensionista de la Universidad Nacional de Avellaneda. ${ }^{4}$ Antropólogo. Docente, investigador y extensionista de la Universidad Nacional de Avellaneda.
} 
la creación de un espacio específico, transdisciplinar, la asignatura Trabajo Social Comunitario.

Es preciso, en primer lugar, situar la construcción de la materia Trabajo Social Comunitario, a través de la cual todos los estudiantes de la UNDAV realizan proyectos de extensión, en el contexto de la universidad pública argentina, especialmente con la creación, en los últimos años, de nuevas universidades nacionales. La Universidad Nacional de Avellaneda (UNDAV) nace al calor de la creación de nueve universidades nacionales en la Argentina, en el período político de reconstrucción nacional que se inició en el año 2003. Desde su Proyecto Institucional, la UNDAV plantea el desarrollo de una Secretaría de Extensión Universitaria que tenga como misión:

Impulsar el acercamiento recíproco y la creación de vínculos de colaboración entre la Universidad y las distintas instituciones gubernamentales y de la sociedad civil en general [...] generando un intercambio que permita no sólo transmitir conocimiento sino ser permeable a recibir aportes que enriquezcan los procesos de enseñanza e investigación considerando el dinamismo de la realidad que obliga a la reflexión constante de los cuerpos teóricos para reformular los paradigmas vigentes, generando y fortaleciendo espacios de diálogo con los diversos sectores de la comunidad que contribuirán a la formación de profesionales comprometidos con las necesidades sociales. ${ }^{5}$ (UNIVERSIDAD NACIONAL DE AVELLANEDA, 2010, p. 132).

Así, nuestra universidad nace bajo el signo de la época: vinculación con el territorio y sus actores sociales constitutivos. Ahora bien, ya no se concibe la relación desde un sentido unilineal, en donde la transferencia y la prestación de servicios son la forma "natural" del compromiso social;

${ }^{5}$ Proyecto Institucional Universitario de la Universidad Nacional de Avellaneda (PIU UNDAV). 
por el contrario, se pone de relieve el carácter dialógico de la articulación. Se visibiliza, así, la trascendencia de las vinculaciones con los actores del territorio ${ }^{6}$ en tanto parte del proceso formativo de estudiantes y docentes.

Frente a la concepción tradicional de la universidad como espacio autónomo, regido por sus propias reglas, se proyectó una institución comprometida con el territorio en el que se inserta. Dicho compromiso ${ }^{7}$, lejos de ser mera enunciación, se ha expresado en prácticas concretas que sitúan a la extensión universitaria, en tanto vinculación con el territorio, a la par de las otras dos funciones fundamentales de la universidad: la docencia y la investigación. Es así que desde su proyecto institucional la universidad ha creado el Trayecto Curricular Integrador Trabajo Social Comunitario. El mismo es concebido en el marco más general de un proceso de vinculación con las organizaciones sociales, en el que se dialoga y se ponen en tensión los saberes académicos. Sin desconocer el rol que la universidad tiene en tanto ámbito legitimado de producción, reproducción y circulación de conocimientos, se trata de resignificar aquellos saberes en el proceso de socializarlos, compartirlos, ampliarlos y enriquecerlos junto con las organizaciones sociales. En esta dinámica los sectores populares no se reducen a versiones más o menos suavizadas de objetivación de un otro extra-universitario. Por el contrario, trabajamos en conjunto con quienes no están dentro de la universidad, consolidando espacios de construcción conjunta de conocimiento. Desde esta perspectiva planteamos que la universidad ya no debe arrogarse un lugar privilegiado en la conducción política de la pro-

${ }^{6} \mathrm{La}$ vinculación entre la universidad y el territorio ha sido planteada de diversas formas, muchas de ellas podrían sintetizarse en la noción de "acoplamiento estructural" (Sturniolo, 2010), es decir, vinculaciones entre dos sistemas cerrados - la universidad, por un lado y la "sociedad" o actores de la misma, por el otro - en donde no se ponen en cuestión las dinámicas propias de lo universitario. Nosotros consideramos al territorio constituido por una trama de actores sociales de la cual la universidad forma parte.

${ }^{7}$ Para una problematización del concepto de compromiso social universitario, y la diferencia con el de responsabilidad social universitaria, así como una historización de las distintas concepciones sobre la vinculación "social" en la universidad argentina, puede verse Cecchi y otros (2009). 
ducción de conocimiento socialmente válido y legitimado, sino que debe producirlo junto a su pueblo ${ }^{8}$.

Es así que los lineamientos de la Secretaría de Extensión Universitaria van de la mano con la creación del Trayecto Curricular Integrador Trabajo Social Comunitario (TSC), teniendo como propósito formar un nuevo tipo de profesional consubstanciado con los intereses de los sectores populares y los movimientos emancipatorios, poniendo en práctica la curricularización de la extensión universitaria. Es decir, integrando la participación en proyectos de Extensión Universitaria como parte de los procesos de enseñanza y aprendizaje de todos los estudiantes de nuestra Universidad. Cabe destacar que tiene un correlato institucional expresado en su texto fundacional:

La UNDAV se constituye como una Universidad con un fuerte compromiso social, por lo que sus actividades de extensión se dirigen a crear y profundizar sólidos vínculos comunitarios. En este sentido se ha establecido para las carreras de pregrado y grado la realización del Trabajo Social Comunitario, cuyo sentido descansa en la convicción de que la Universidad debe generar en el estudiante y futuro profesional la responsabilidad de involucrarse como actor social, y que sus saberes acompañen los procesos de cambio. (UNIVERSIDAD NACIONAL DE AVELLANEDA, 2010, p. 136).

${ }^{8}$ Entendemos que el concepto de pueblo no es unívoco ni está exento de contradicciones; de hecho, quizás sea más pertinente para dar cuenta del proceso histórico de conformación del estado-nación en la Argentina hablar de "clases populares". No obstante, creemos necesario reivindicar la categoría pueblo para pensar y actuar un modelo de universidad que se desarrolle tomando posición y dando cuenta de las asimetrías sociales que nos atraviesan. La categoría pueblo nos interpela al menos desde dos direcciones, por un lado en tanto pone de relieve la cuestión nacional a partir de su entrelazamiento con los sujetos colectivos; por otra parte, en tanto da cuenta de las contradicciones internas e intereses divergentes entre los distintos sectores sociales que pugnan por el control del aparato estatal. Para una discusión en torno al desarrollo de las clases populares en la Argentina véase ADAMOVSKY, Ezequiel. Historia de las clases populares en la Argentina. Desde 1880 a 2003. Buenos Aires: Sudamericana, 2012. 
Ahora bien, es preciso situar la propuesta de la universidad en el escenario nacional, en tanto expresión de un proceso social e histórico. La universidad argentina se ha caracterizado por su masividad y "gratuidad" (no arancelamiento). No forma parte de este trabajo hacer un relato pormenorizado de su desarrollo en el último siglo, pero si es preciso destacar que el mismo fue transformándose al calor de la vida política y social de la Argentina. Podemos señalar algunos puntos de inflexión, tomando como factor común la democratización y el cuestionamiento de su rol social: la Reforma del 18 durante el primer gobierno de Irigoyen (primer presidente electo con voto masculino universal y secreto); el no arancelamiento e ingreso irrestricto durante el primer gobierno peronista"; la breve "primavera" de 1973, momento de fuerte activismo político y social en la universidad, previo al golpe militar de 1976; y, por último, el actual período histórico, en el que se ha ampliado fuertemente la matrícula universitaria, ingresando de forma masiva a las universidades estudiantes que son la primera generación de universitarios en sus familias.

Esta característica del no arancelamiento vigente a partir de un decreto del primer gobierno del Gral. Juan Domingo Perón en 1949 resulta ineludible para comprender la conformación del sistema Universitario argentino. La historiografía oficial no ha dado cuenta significativamente de este hecho, subrayando sólo como acto fundante la reforma de 1918. A estos dos momentos constitutivos de nuestro sistema universitario, es preciso incorporar un tercer momento iniciado a partir del repliegue de las políticas neoliberales en el año 2003. A partir del proceso político encabezado por el entonces presidente Néstor Kirchner, las universidades estatales atraviesan un tiempo de mucha potencia, en el que la matrícula universitaria ha crecido considerablemente y en el que se han creado 14 nuevas universidades en todo el país, permitiendo que en todas las provincias haya al menos una Universidad pública, no arancelada y de acceso irrestricto. El

${ }^{9}$ Para una historia de la universidad argentina, especialmente en el período del primer gobierno peronista, ver: RECALDE, Aritz; RECALDE, Iciar: Universidad y Liberación Nacional: Un Estudio de la Universidad de Buenos Aires Durante Las Tres Gestiones Peronistas: 1946-1952, 1952-1955 y 1973-1975. Buenos Aires: Buenos Tiempos, 2007. 
ejercicio del derecho a la educación superior es acompañado además por diversos programas de becas que permiten apuntalar los deseos de estudiar de los sectores populares y trabajadores.

Cualquier propuesta respecto a pensar el rol de la Universidad, el lugar que la misma debe cumplir en la sociedad no puede sustraerse del enorme esfuerzo que hace el pueblo argentino en sostenerla. Es por eso que consideramos que estamos ante una oportunidad histórica, que es la de construir una universidad comprometida con la suerte de su pueblo y de su nación. Es en este escenario que debe comprenderse la vinculación sociocomunitaria en tanto práctica formativa.

En el contexto ${ }^{10} \mathrm{y}$ a partir de las características del sistema universitario argentino, el derecho a la educación superior no puede considerarse un derecho individual sino que debe pensarse como un derecho colectivo. Es decir que: no resulta suficiente que el estudiar en la universidad ya no sea el privilegio de las clases medias, sino que además el conjunto del pueblo y la nación deben ser destinatarios del quehacer de dicha institución pública.

\footnotetext{
${ }^{10} \mathrm{Al}$ momento de revisar estas líneas, promediando el 2016, muchas de las condiciones descriptas en el artículo están siendo cuestionadas fuertemente por las políticas del gobierno nacional y, por otra parte, por el creciente deterioro de la situación socioeconómica de amplios sectores de la población. El poder adquisitivo de los asalariados argentinos ha disminuido significativamente y han aumentado los índices de desempleo. Se estima que al menos un $9 \%$ de la matrícula de la UNDAV ha abandonado sus estudios en el primer semestre de 2016. De esta forma, dramáticamente, vuelve hacia la institución universitaria la pregunta-problema en torno de lo popular. ¿Qué rol debe jugar una universidad nacional en un escenario de creciente conflictividad social? ¿Qué hacer cuando la propia comunidad universitaria se ve vulnerada por la situación social? Creemos que en contextos complejos como el actual, queda en evidencia más que nunca que no puede pensarse a la universidad por fuera de la sociedad de la que forma parte.
} 


\section{UNA PROPUESTA CONCRETA: EL TRAYECTO CURRICULAR INTEGRADOR TRABAJO SOCIAL COMUNITARIO}

La voluntad y el deseo de estrechar los vínculos con los actores extra universitarios y comprometer a la universidad con los complejos escenarios de la realidad social nos exige encontrar y crear formatos concretos que permitan y expresen dicha relación. En el mundo de la universidad, los contenidos se realizan en los planes de estudio: su pertinencia o necesidad, en definitiva, su importancia en la formación de los futuros profesionales se ve reflejado en la currícula.

Claro está que su inclusión en los programas, en los tiempos concretos y finitos de las cursadas, implica inevitablemente una operación de recorte, de reducción. Somos conscientes de que solamente una asignatura concreta no puede dar cuenta de la diversidad de saberes, conocimientos, experiencias de organización y gestión que se construyen desde lo popular; y no sería coherente, además, reducir la integración de la universidad, en sus distintas funciones, con la compleja trama de lo social a un único espacio.

Ahora bien, la existencia de espacios curriculares en donde se dialoga con los actores del territorio, posibilitándose interacciones directas - rompiendo con la lógica hegemónica en nuestra sociedad de la realidad mediatizada, de la percepción colonizada por los dispositivos masivos de comunicación - es un punto de partida para hacer una universidad popular.

Es así que el trayecto consta de cuatro asignaturas, en términos generales, la modalidad de cursada de los niveles I, II, III y IV es cuatrimestral y consiste en dos horas semanales, contabilizando treinta y dos horas por cada nivel.

En el primer nivel (TSC I), la modalidad de trabajo se desarrolla en el aula y hace hincapié en el abordaje político-pedagógico del Trayecto, 
para luego ahondar, desde el enfoque de las ciencias sociales, en las discusiones sobre la canónica dicotomía objetividad/subjetividad de la ciencia. Luego, se hace foco en el rol de los medios de comunicación, en los imaginarios sociales y en las construcciones de sentido común que atraviesan actualmente la sociedad, para contraponer a la concepción positivista del conocimiento la teoría dialéctica y el paradigma de la multiperspectividad. Se trabajan, entonces, casos concretos vinculados a problemáticas socioambientales que posibiliten el debate y la reflexión desde diferentes miradas.

En el segundo y tercer nivel (TSC II y TSC III) los estudiantes participan de proyectos de extensión - presentados por docentes de la universidad - que son desarrollados en el territorio y se articulan, al mismo tiempo, con herramientas teórico-metodológicas a través de encuentros en el aula. Si bien los encuentros se dividen equitativamente, lo cierto, y lo interesante sobre todo, es que estos dos espacios tienden a borrarse. En estos años, en algunos proyectos los encuentros "de aula" se realizaron en la organización, con participación de sus integrantes.

Ahora bien, resulta necesario establecer algunas diferencias entre los enfoques de ambos niveles. El segundo nivel propone el desarrollo de un proyecto de extensión universitaria que se diseña y se lleva a cabo bajo la coordinación de un docente de la UNDAV, mediante un acuerdo conjunto y una redefinición constante con referentes de instituciones gubernamentales y organizaciones sociales, políticas y culturales con anclaje en el territorio. En esos proyectos se apunta, fundamentalmente, a que los estudiantes se aproximen a las categorías de problema social y política pública, en relación con alguna experiencia comunitaria que permita, a su vez, problematizar y poner en debate las formas de participación y los modelos de planificación y programación social. De esta manera, resulta central en los proyectos de este nivel construir, junto con las organizaciones, un mapa colectivo acerca del territorio en el cual se ubica la organización, conocerlo, caminarlo, y dialogar con los sujetos que allí habitan, y con los referentes comunitarios que llevan adelante la organización. En ese recor- 
rido la propuesta implica - necesariamente - la incorporación de docentes y estudiantes a la dinámica del espacio, a su cotidianeidad, para empezar a conocer sus representaciones, sus ideas, así como trabajar los propios prejuicios acerca de ese otro al que, en un primer momento, desconocemos y que, en ese proceso, empezamos a conocer a partir del trabajo concreto y del compartir cotidiano. Los proyectos pueden implicar o bien la profundización de alguna línea de trabajo ya iniciada por la institución u organización, o bien el comienzo de un proyecto que a la vez posibilite el proceso pedagógico que nos proponemos que atraviesen los estudiantes. Para esto, los alumnos deben llevar adelante, en cada uno de los proyectos, registros etnográficos y entrevistas diversas de lo que acontece en su participación en el territorio. Este encuadre metodológico permite trabajar en profundidad sobre el eje reflexivo del trayecto a la vez que afianza vínculos con los vecinos. Por su parte, las entrevistas, permiten socializar información con la organización de modo serio y sistematizado.

El tercer nivel de Trabajo Social Comunitario trabaja junto con organizaciones sociales y políticas a través de proyectos que tienen como eje metodológico la Investigación Acción Participativa (IAP) ${ }^{11}$. Dicha propuesta supone dos procesos que entendemos interrelacionados: por un lado, la participación de los sectores populares a través de sus organizaciones - sobre los procesos de producción, validación y pertinencia de los conocimientos generados en la universidad pública. Por otro, las acciones y transformaciones que los nuevos conocimientos permiten desplegar. La propuesta requiere de ambos pares.

Este tercer nivel propone, además, una aproximación participativa al acontecer de las organizaciones propias de los sectores populares. Son fundamentales, para esto, sus avances y retrocesos, su vida cotidiana,

\footnotetext{
${ }^{11}$ Para una discusión en torno a la propuesta de la Investigación Acción Participativa ver: FALS BORDA, Orlando. La investigación participativa y la intervención social. En: Documentación social. Revista de estudios sociales y sociología aplicada, n. 92, jul./sep. 1993. Para el desarrollo metodológico de la misma: ANDER-EGG, Ezequiel. Repensando la Investigación Acción Participativa. Buenos Aires: Lumen Humanitas, 2003.
} 
los cambios culturales e identitarios, los vaivenes ideológicos y las formas de organización y acción política desplegadas en los distintos momentos socio-históricos que han atravesado hasta llegar al presente. La reconstrucción de la historia de los sectores populares se acompaña con la asistencia bibliográfica de la materia y en vinculación con una organización popular concreta. Dicha reconstrucción supone una producción colectiva entre docentes, estudiantes y las organizaciones protagonistas. En tal sentido, en este nivel, los sujetos protagonistas son las organizaciones sociales y políticas; es por eso que se articula con Proyectos de Extensión Universitaria cuyo objetivo es la reconstrucción de la memoria oral de dichas organizaciones populares. El proceso deriva en acciones conjuntas y diversas como: talleres, folletos, charlas, radios abiertas, organización de eventos o campañas, etc. A su vez, hacia el final del proyecto la propuesta incluye pensar junto con las organizaciones - luego de conocer su historia, devenir, problemáticas y contexto actuales - posibles líneas de acción a futuro que puedan tomar forma de nuevas actividades, programas o proyectos de extensión a desarrollarse en las organizaciones con las que se ha participado.

Siempre con la propuesta de reflexionar y conceptualizar en torno a la experiencia de procesos de organización y participación social, el tercer nivel de Trabajo Social Comunitario procura que dicha praxis brinde un material que permita contextualizar (histórica, social, económica, cultural y políticamente) las situaciones abordadas con las organizaciones y sectores sociales donde se llevan adelante los proyectos de extensión. Estas reflexiones permiten deliberar sobre los avances y retrocesos de los sectores populares y sus organizaciones. El desafío del tercer nivel pasa por producir conocimiento desde un enfoque de Investigación Acción Participativa e implica poder trabajar en conjunto con organizaciones del campo popular. A su vez, se historiza y se pone en tela de juicio el concepto de extensión universitaria, planteado en la dicotomía señalada por Freire - comunicación o extensión - (FREIRE, 1973), para reflexionar en torno al vínculo entre la universidad y los profesionales y la sociedad de la que forman parte. 
El cuarto nivel, por su parte, apunta al diseño de proyectos sociocomunitarios por parte de los estudiantes, en conjunto con las organizaciones e instituciones con las que trabajaron en los niveles II y III, o bien con actores que los propios estudiantes contactan. Aquí la propuesta vuelve a reunirlos por departamentos y disciplinas para pensar proyectos específicos en temáticas afines a la carrera que se encuentran estudiado. La aprobación de este último nivel supone, por tanto, el diseño de un proyecto de extensión que pasará a integrar el Banco Universitario de Proyectos de Extensión (BUNIPE).

En este último nivel de TSC, la propuesta interpela directamente a la formación académica de los estudiantes. Con base en la apropiación de conocimientos teóricos, prácticos y metodológicos desarrollados durante la carrera, y con el recorrido de los niveles I, II y III de TSC, el planteo desafiante y enriquecedor es que puedan posicionarse desde esa formación. Es interesante destacar aquí que, de alguna manera, este cuarto nivel da respuesta a una demanda que plantean los estudiantes desde el primer cuatrimestre en que cursan: la de poder aportar desde sus disciplinas a la transformación del territorio. Mientras que en los primeros niveles se pone énfasis en la desnaturalización de la realidad social, cuestionando nuestros propios presupuestos, invirtiendo el orden del binomio universidad-territorio, para reconocer a los saberes populares en su validez y comprensión del mundo ${ }^{12}$; en el cuarto nivel el protagonismo está puesto en los y las estudiantes, quienes, a partir del diálogo, tienen la responsabilidad de poner en juego su recorrido universitario. Tanto el disciplinar como la labor extensionista desarrollada en el marco de la materia TSC.

Siguiendo la línea de los anteriores niveles, el requisito para los proyectos de extensión que se diseñan es que articulen siempre con al menos una organización o institución contraparte que se comprometa con el desarrollo del proyecto. Basados en la experiencia de los primeros proyec-

\footnotetext{
${ }^{12}$ Podríamos sintetizar esto diciendo: nuestros docentes son las organizaciones sociales, nosotros, como universidad, vamos a aprender del y en el territorio, a partir de las experiencias anteriores a nosotros $\mathrm{y}$, fundamentalmente, a partir de un quehacer compartido.
} 
tos que venimos desarrollando, consideramos fundamental subrayar este aspecto. Todos los proyectos de extensión que se integran a Trabajo Social Comunitario deben diseñarse y llevarse a cabo en conjunto con instituciones gubernamentales y no gubernamentales u organizaciones sociales, políticas y culturales, con las que se articule el trabajo concreto en el marco de los proyectos y que, por lo tanto, tengan una inserción territorial genuina y sostenida en el tiempo. Sólo puede darse un diálogo de saberes entre sujetos, y la universidad, en tanto actor institucional, debe reconocer los agrupamientos y construcciones colectivas del territorio.

\section{ACERCA DE LA INCLUSIÓN EN LA CURRÍCULA DE UN TRAYECTO INTEGRADOR: LA OBLIGATORIEDAD COMO RECONOCIMIENTO DE UNA NECESIDAD FORMATIVA}

Cuando se reflexiona en torno a la curricularización de la extensión universitaria uno de los debates giran en torno a la "obligatoriedad" o "voluntariedad" de las prácticas. Esta discusión se circunscribe - muchas veces - de tal forma que se piensa la integración de la praxis social a la currícula por fuera de la planificación estratégica de una carrera, como si las carreras de la mayor parte de las Universidades no se conformaran con un amplio porcentaje de materias que no son optativas, sino que han sido fijadas como fundamentales para el desarrollo del futuro profesional. Toda carrera universitaria, como todo desarrollo científico - y no solo la curricularización de la extensión - descansa en principios que se asumen por acción u omisión, son parte de una decisión política que es la síntesis de debates y disputas respecto a qué tipo de profesional debe formar la universidad pública. La propuesta de curricularización implica un proceso de reflexión-acción-reflexión, en el que se reorganiza la relación entre la construcción del conocimiento y la acción transformadora, entre el sujeto y el objeto. Por lo tanto, creemos que no puede ser algo anexo o anecdótico sino que el integrar la acción transformadora al interior de los procesos 
de reflexión - implicando insertar elementos dinamizadores, muchas veces contradictorios, que puedan tensionar la pertinencia de la ciencia y de los procesos de construcción de conocimiento - es un elemento esencial e impostergable que deben asumir los procesos de formación de nuestras Universidades.

Es en este sentido que entendemos que la integración de la extensión a los procesos formativos en nuestras universidades encierra discusiones respecto a la forma en que se diseñarán las propuestas para cada casa de estudios. Estas discusiones nos parecen tan válidas como necesarias, pero creemos esencial clarificar el objetivo político que implica ligar la enseñanza y la construcción de conocimiento a la praxis extensionista.

\section{LA EXTENSIÓN UNIVERSITARIA COMO ARTICULADOR POLÍTICO TERRITORIAL}

Es preciso dejar de considerar a la extensión como un mero complemento, secundario o eventual, de las áreas consideradas históricamente como principales: la investigación y la docencia. Abandonar la idea de la Universidad en tanto enclave colonial, con el deber de irradiar "el saber" y la "alta cultura" sobre las clases subalternas de los países dependientes. Dejar de entenderla como un estrecho canal a través del cual nos relacionamos con el resto de los actores sociales, debe implicar reconfigurar su rol en términos de pensar a la extensión como un articulador político-territorial que interpele la formación de futuros profesionales y permita ser un constructor de demanda que defina líneas de investigación al servicio de los sectores populares y el desarrollo nacional. Investigación, docencia y extensión no pueden ser pensados aisladamente por quienes insistimos en que debemos discutir nuevos modos de hacer universidad. Integrar dichas funciones significa generar un modelo de universidad que, abierta y flexible, se estructure a partir de las problemáticas sociales, dejando de usar 
a la realidad como mera excusa para su autorreproducción. Pero además, partiendo de la no neutralidad de la educación, consideramos fundamental ligar, a través de los espacios y procesos de vinculación que abre la extensión universitaria, la educación superior a las luchas sociales y políticas emancipadoras protagonizadas por los movimientos populares. Las múltiples interacciones entre políticas sociales, instituciones y actores, contextualizadas territorial e históricamente, pueden constituir entonces los objetos de conocimiento y de intervención, y ser los ordenadores de propósitos y contenidos a enseñar y aprender. Esto, además de posibilitar una mayor imbricación de la universidad con el territorio del que forma parte, debe contribuir a combinar los intereses nacionales y populares - en tiempo de profundas transformaciones como las que viven varios de los países latinoamericanos - con los de la formación y la investigación.

Concebir al rol de la extensión como un articulador político-territorial nos concede la posibilidad de no limitar la intervención en el territorio en términos técnicos sino entenderla como teórica, política y posicionada en el marco de las disputas que existen en dicho campo. Lo antedicho nos invita a considerar que los debates respecto de cuál es la dosis necesaria de horas extensionistas es subsidiaria al debate de cómo articulamos mejor la enseñanza y la investigación que permita poner a los futuros profesionales y al conocimiento científico al servicio de la transformación de la sociedad.

Las universidades y los universitarios no podemos limitar nuestro quehacer académico a "la crítica" y "la denuncia" entre convencidos, sino que ante la injusticia social es imprescindible elaborar - construir - procesos de enseñanza y construcción de conocimiento en movimiento, es decir, orgánicos a los procesos de lucha por la emancipación nacional y social.

Curricularizar la extensión Universitaria implica para nosotros la apertura de un proceso de aprendizaje en movimiento que busca incorporar experiencias y conocimientos sociales populares, muchas veces silenciados, marginados y combatidos por la epistemología oficial. Una propuesta 
de diálogo de saberes flexible a oír otras formas de conocer reconociéndolas como parte de una creatividad popular repetidas veces subalternizadas por la razón colonizadora europea.

No hay liberación nacional y social posible recetada desde afuera de la nación y externa a las clases populares. Y la universidad en Latinoamérica compromete alterar una profunda temporalidad histórica y simbólica de sedimentación colonial. La dominación colonial no sólo ha sido económica, política o administrativa sino que ha atravesado nuestros cuerpos, nuestros pensamientos, es decir, nuestras subjetividades. La colonialidad ha consistido en el menosprecio de lo propio (lo indígena, africano, mestizo, nacional, latinoamericano) generando un vaciamiento de la subjetividad del colonizado, incapaz de pensar que las soluciones a sus problemas encontrarán caminos más significativos en autores nacionales y experiencias populares que en la importación de fórmulas para problemas ajenos. El aprendizaje en movimiento no es una mera invitación a un pluralismo de saberes carente de intencionalidad política, sino, por el contrario, es una propuesta que busca sacudir las estructuras colonizadas del pensamiento, para ser parte de los procesos de lucha política emancipatoria.

Por otra parte, el aprender en movimiento involucra no sólo debates que consideren los enfoques multidisciplinares, interdisciplinares y transdisciplinares capaces de quebrar las fronteras disciplinares, interpelando el modelo tecnicista del conocimiento y capaces de evitar la fragmentación de las interpretaciones y de las experiencias, sino que, además, los proyectos desarrollados bajo una perspectiva dialógica habilitan también enfoques multisectoriales que fuerzan a los procesos de enseñanza, aprendizaje y producción de conocimiento, a "[...] buscar afuera de nuestros paradigmas, enfoques, disciplinas y campos de conocimiento."(CASTRO-GÓMEZ, 2007, p. 17). Rastreando entre los saberes populares que fueron forjados, entre las organizaciones sociales, políticas, en ámbitos religiosos, étnicos, asociativos y situados - casi siempre - en contextos de resistencia cultural, de lucha por la igualdad o el reconocimiento, los enfoque 
multisectoriales tienen el desafío de integrar a los habituales damnificados por un colonialismo que nunca limitó su dominación al plano político y económico sino que ha efectuado una sistemática invisibilización epistémica, cuando no verdaderos epistemicidios, sobre los saberes de los sectores populares en los países periféricos.

\section{APRECIACIONES FINALES}

Creemos firmemente en la potencialidad de un espacio de trabajo interdisciplinario como ámbito formativo y de vinculación con los actores del territorio. O dicho de otra forma, es importante, al decir del uruguayo Gabriel Kaplún, indisciplinar la universidad (KAPLÚN, 2003): si la universidad se construyó desde sus orígenes como el lugar de lo unívoco, del saber legitimado, que a su vez se proyectaba sobre el "exterior" justificando un orden social desigual y estamentario, la posibilidad de guiar la construcción del conocimiento a partir de la realidad social, a partir de la perspectiva de los actores, nos pone en el lugar de producir una democratización del conocimiento, no en su consumo, sino fundamentalmente en su elaboración.

Si bien las distintas materias de todas las carreras tienen que hacer experiencias de extensión, ${ }^{13}$ en nuestra breve experiencia hemos podido apreciar que un espacio de enseñanza aprendizaje en donde se rompe con la normalidad de la disciplina es muy valorado por los estudiantes, quienes destacan, además, la posibilidad de vincularse con compañeros y compañeras de otras especialidades, con lo que significa la riqueza de las distintas miradas, que ayudan a tener una visión compleja de la problemática trabajada, en donde cada estudiante aporta desde su lugar. Trabajo Social Comunitario es un trayecto profundamente formativo, en donde toda experiencia es un hecho pedagógico, ese aprender haciendo, incluso en los

${ }^{13}$ Parafraseando el sentir general de los participantes del Foro de Extensión del Mercosur, realizado en la ciudad de Passo Fundo en agosto del 2015, "tenemos que ir por el cien por ciento". 
fracasos, en lo que no se logra, nos muestra la complejidad de la realidad social. En tanto propuesta de construcción colectiva del conocimiento nos tensiona, permanentemente, con la necesidad y la demanda institucional de la evaluación y la calificación, en donde no se puede perder de vista tanto la importancia del proceso como del resultado.

La inclusión en la currícula de la extensión, se trata de un debate político: vincularse con los actores sociales no es en sí mismo bueno o malo. La raíz de la discusión estriba en si, como universitarios, como institución universidad, nos reconocemos en un entramado social y en una historia: la universidad argentina es el resultado de luchas históricas por su democratización, el trabajo en el territorio se plantea desde una perspectiva de restitución de derechos vulnerados, se trabaja junto a actores que han sido protagonistas en pelear por sus derechos, se acompaña la política pública que da respuesta a una necesidad.

Si pensamos que la extensión universitaria es una herramienta para la transformación social, no podemos dejar de problematizar el rol de la universidad como institución, cuál es su especificidad: la construcción de conocimiento y su reproducción, la formación de futuros profesionales, que actuarán en la sociedad. Sólo puede tener un carácter transformador en la medida en que se vincula con la política pública, con el estado - reconociéndonos también dentro de la compleja trama institucional que es el estado - y las organizaciones del pueblo ${ }^{14}$.

A modo de cierre, nos interesa subrayar que aquello que llamamos aprendizaje en movimiento implica concebir el conocimiento como una acción. Implica, por tanto, sostener que el conocimiento no es una reproducción o simple reflejo de las cosas, no entendemos al conocimiento como meras imágenes mentales sobre una realidad que es externa al sujeto

\footnotetext{
${ }^{14}$ Hemos apelado permanentemente, a lo largo del artículo, a la categoría "pueblo" y "popular". Complementando la aclaración hecha anteriormente, entendemos que es preciso recuperar dicho concepto, en toda su complejidad y heterogeneidad; de hecho, la práctica concreta es la que nos permite definirlo, cuestionarlo, pensarlo, hacerlo real en cuanto a su incidencia en la universidad.
} 
cognoscente. No entendemos al conocimiento como mera contemplación, es decir, no lo concebimos desligado de la actividad humana, pues creemos que el hombre sólo conoce en cuanto crea la realidad humana y social en que está inmerso. La praxis es propiamente la esfera del ser humano. Sin praxis no hay realidad humana, y sin ella no hay tampoco conocimiento del mundo. "Para que el mundo pueda ser explicado críticamente es necesario que la explicación sea situada en el terreno mismo de la praxis revolucionaria” decía el autor checo Kosic (1967, p. 13). La tan mentada, muchas veces, unión entre teoría y práctica encuentra su articulación en la praxis que el hombre realiza en el mundo histórico-social, un mundo construido, producido y reproducido por el ser humano. La amalgama entre teoría y práctica no puede ser imaginada en una realidad abstracta, pura o autónoma de tensiones políticas en las que se desenvuelve dicha praxis humana, sino por el contrario, la misma se realiza en un territorio político repleto de contradicciones, intereses y proyectos políticos (implícito o explícito) diferente y/o antagónico. La propuesta de aprendizaje en movimiento explicita tanto el carácter político de la educación, como la vinculación estratégica y el rol protagonista de los movimientos populares para tensionar las visiones reproductivistas del conocimiento que presentan la realidad como algo dado o a ser resuelto por elites iluminadas, es decir, un conocimiento tecnicista, una historia de "próceres" y no los procesos históricos que protagonizan los pueblos. No hay transformación posible sin protagonismo popular.

\section{REFERENCIAS}

ADAMOVSKY, E. Historia de las clases populares en la Argentina. Desde 1880 a 2003. Buenos Aires: Sudamericana, 2012.

ANDER-EGG, E. Repensando la Investigación Acción Participativa. Buenos Aires: Lumen Humanitas, 2003. 
ÁVILA HUIDOBRO, R. et al. Universidad, territorio y transformación social: reflexiones en torno a procesos de aprendizaje en movimiento. Avellaneda: UNDAV Ediciones, 2014.

CASTRO-GÓMEZ, S. El giro decolonial: reflexiones para una diversidad epistémica más allá del capitalismo global. Buenos Aires: Siglo del Hombre Editores, 2007.

CECCHI, N. et al. El compromiso social de la universidad latinoamericana del siglo XXI: entre el debate y la acción. Buenos Aires: IEC, 2009.

FALS BORDA, O. La investigación participativa y la intervención social. En: Documentación social. Revista de estudios sociales y sociología aplicada, n. 92, jul./sep. 1993.

FREIRE, P.¿Extensión o comunicación? La concientización en el medio rural. Buenos Aires: Siglo XXI, 1973.

KAPLÚN, G. Indisciplinar la universidad. Quito: Universidad Andina Simón Bolívar, 2003.

KOSIC, K. Dialéctica de lo concreto: estudio sobre los problemas del hombre y del mundo. México: Grijalbo, 1967.

RECALDE, A.; RECALDE, I. Universidad y Liberación Nacional: Un Estudio de la Universidad de Buenos Aires Durante Las Tres Gestiones Peronistas: 1946-1952, 1952-1955 y 1973-1975. Buenos Aires: Buenos Tiempos, 2007.

STURNIOLO, S. Inflexiones en el discurso académico: la universidad y su articulación local. Revista del Instituto de Investigación en Ciencias de la Educación, Buenos Aires, año XVII, n. 28, 2010.

TOMMASINO, H. Generalización de las prácticas integrales: los aportes de la extensión para su implementación. Diálogo, Montevideo, año 1, n. 3, 2008 . 
UNIVERSIDAD NACIONAL DE AVELLANEDA. Proyecto Institucional Universitario de la UNDAV. Avellaneda, Buenos Aires, Argentina: UNDAV, 2010, p. 132, 136. 